\title{
Role of Thermal Equilibrium Dynamics in Atomic Motion during Nonthermal Laser-Induced Melting
}

\author{
Xiaocui Wang $\odot,{ }^{1}$ J. C. Ekström, ${ }^{1}$ Å. U. J. Bengtsson, ${ }^{1}$ A. Jarnac, ${ }^{2,3}$ A. Jurgilaitis $\odot,{ }^{2}$ Van-Thai Pham, ${ }^{2}$ \\ D. Kroon $\odot,{ }^{2}$ H. Enquist, ${ }^{2}$ and J. Larsson $\oplus^{1,2, *}$ \\ ${ }^{1}$ Department of Physics, Lund University, P.O. Box 118, SE-221 00 Lund, Sweden \\ ${ }^{2}$ MAX IV Laboratory, Lund University, P.O. Box 118, SE-221 00 Lund, Sweden \\ ${ }^{3}$ ONERA, Université Paris Saclay, F-91123 Palaiseau, France
}

(Received 16 January 2020; revised manuscript received 13 February 2020; accepted 18 February 2020; published 12 March 2020)

\begin{abstract}
This study shows that initial atomic velocities as given by thermodynamics play an important role in the dynamics of phase transitions. We tracked the atomic motion during nonthermal laser-induced melting of InSb at different initial temperatures. The ultrafast atomic motion following bond breaking can in general be governed by two mechanisms: the random velocity of each atom at the time of bond breaking (inertial model), and the forces acting on the atoms after bond breaking. The melting dynamics was found to follow the inertial model over a wide temperature range.
\end{abstract}

DOI: 10.1103/PhysRevLett.124.105701

Melting processes are of fundamental interest and of technological importance, as evidenced by melt-induced permanent phase transitions used in optical recording and phase-change memory devices [1]. Ultrafast melting is a field of intense study, both experimentally $[2,3]$ and theoretically [4,5], in which the dynamics of melting reveal the underlying physics governing the process, which differs depending on the type of material that is studied. In laserinduced melting of metals, the light interacts with electrons in the conduction band, and the energy is subsequently transferred to the lattice through electron-phonon coupling. This lattice heating can result in thermal melting, which occurs on the ps timescale $[3,6]$. A process similar to thermal melting occurs in semiconductors at low laser fluences. In contrast, nonthermal melting of semiconductors at high laser fluences is associated with bond breaking, resulting in the loss of cohesion between the atoms in the crystal [7].

The study of nonthermal melting started in 1979 when Van Vechten et al. [8] investigated laser annealing of silicon using nanosecond pulses, and predicted that melting was not governed by thermal processes. Shank et al. [9] later discovered that the optical reflection coefficient changed from that of the solid to that of the liquid within a few 100 fs. The first structural studies were based on a symmetry change in the signal resulting from optical second harmonic generation when the crystal structure

Published by the American Physical Society under the terms of the Creative Commons Attribution 4.0 International license. Further distribution of this work must maintain attribution to the author(s) and the published article's title, journal citation, and DOI. Funded by Bibsam. was lost [10], and the first studies using $\mathrm{x}$ rays showed that the structural disordering occurred at depths of tens of $\mathrm{nm}$ $[11,12]$. By 2005, the signal-to-noise ratio and the temporal resolution obtainable in such studies had become sufficiently good to allow the determination of the temporal shape of the x-ray scattering signal during nonthermal melting of the III-V semiconductor InSb [2]. The resulting Gaussian shape, together with the relationship between the timescale of the decrease in the scattered $\mathrm{x}$-ray intensity for two different Bragg reflections led Lindenberg et al. to formulate the inertial model [2]. This model describes the temporal evolution of nonthermal melting of a semiconductor. The idea behind the model is that the unbound atoms move at the random velocity they had at the time when the bonds were broken. This model has been disputed by Zijlstra et al. [5] based on ab initio time-resolved density functional theory (DFT) calculations. The authors concluded that the atomic motion was instead governed by forces acting on the atoms after bond breaking.

Understanding the physics governing nonthermal melting remains the subject of considerable interest $[4,5,13,14]$. In this study, we used ultrafast x-ray diffraction (XRD) to investigate the importance of the initial atomic velocities given by thermal equilibrium dynamics. We carried out experiments under low laser excitation conditions, thereby reducing the material-specific accelerating forces. By changing the initial temperature before laser excitation we were able to control the initial velocities and measure the disordering time. We show that the timescale of a phase transition can be influenced by the initial equilibrium temperature before laser excitation. The measured timescales are in very good agreement with the inertial model over the temperature range studied. 
The time dependence of $x$-ray diffraction signals in structural studies allows the timescale of a phase transition to be determined. Here, we refer to the time taken for the diffracted x-ray intensity to fall from $90 \%$ to $10 \%$ of the initial value as the "disordering time." The inertial model [2] allows the disordering time to be modeled by determining the atomic velocity at thermal equilibrium using the equipartition theorem. The Debye-Waller formalism can then be applied to determine the time-resolved x-ray intensity $I(t)$ scattered from an ensemble of atoms in which the individual atoms are moving in different directions:

$$
I(t)=I(0) e^{-q^{2}\left\langle u^{2}(t)\right\rangle / 3},
$$

where $q$ denotes the magnitude of the momentum transfer vector, and the average mean square displacement is given by

$$
\left\langle u^{2}(t)\right\rangle=v_{i}^{2} t^{2},
$$

where $t$ denotes time.

The initial (root-mean square) velocity is given by

$$
v_{i}=\sqrt{2 U_{\mathrm{Kin}} / M},
$$

where $M$ is the atomic average mass. According to the virial theorem, the average kinetic $\left(U_{\mathrm{Kin}}\right)$ and potential energy $U_{\text {Pot }}$ are equal

$$
U_{\text {Kin }}=U_{\text {Pot }}=U / 2 .
$$

The thermal vibrational energy $(U)$ of a material [15] is given by

$$
U=\frac{9}{8} k_{B} T_{D}+3 k_{B} T D_{3}\left(T_{D} / T\right),
$$

where $T$ is the temperature, $k_{B}$ is the Boltzmann constant, and $T_{D}$ is the Debye temperature which is $160 \mathrm{~K}$ for $\mathrm{InSb}$. $D_{3}(x)$ is the Debye function which at high temperatures approaches 1, and the classical result of

$$
U=3 k_{B} T
$$

is recovered. In the low temperature limit, it can be seen that Eq. (5) approaches $U=\frac{9}{8} k_{B} T_{D}$, which is the zeropoint vibrational energy.

In theoretical studies [4,5,16], the initial velocity is ignored, and the atomic velocities are obtained only from the acceleration of the atoms along the modified potential landscape. Time-resolved DFT calculations give the forces acting on the ions as the derivative of the free electron energy, used to predict the atomic velocities for different laser energy densities.

To investigate the importance of inertial motion in determining the timescale of a phase transition, we have measured the disordering time for nonthermal melting of $\mathrm{InSb}$ for a series of initial temperatures using ultrafast $\mathrm{XRD}$. One would expect the disordering time not to vary with temperature if the initial velocities can be neglected. However, the disordering time should vary and be dictated by Eqs. (1)-(5) if the disordering process is dominated by the initial velocities. In the classical limit, the disordering time would scale inversely with the square root of the temperature. To visualize the structural dynamics and calculate the scattered $\mathrm{x}$-ray intensity during nonthermal melting obeying inertial motion, we performed molecular dynamics (MD) simulations (Fig. 1). We used the wellestablished open-source software, large-scale atomic or molecular massively parallel simulator (LAMMPS), to obtain the initial velocities used as input for the inertial model after laser excitation. The calculations were performed using a cell size of $8 \times 8 \times 8$ unit cells, each containing 8 atoms, although only 18 of the 4096 atoms are shown in Fig. 1. Periodic boundary conditions were applied. The simulations were carried out using the empirical potential proposed by Shimojo et al. [15]. The coefficients describing the potential for InSb were provided (a)

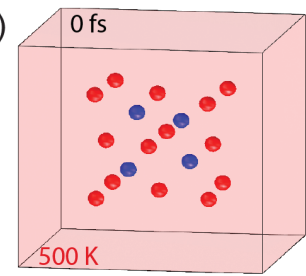

(c)

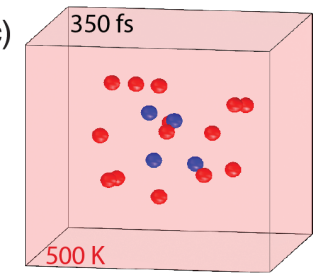

(e)

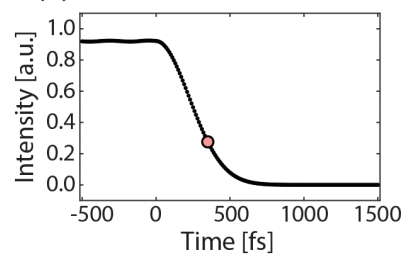

(b)

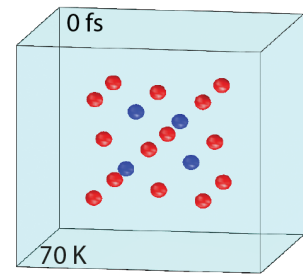

(d)

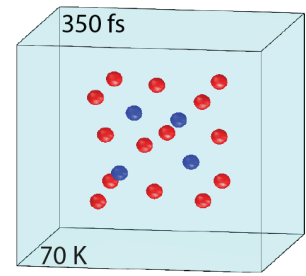

(f)

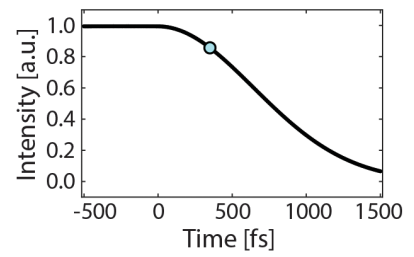

FIG. 1. Illustration of the dynamics of laser-induced melting of $\mathrm{InSb}$ when governed by thermal equilibrium dynamics. The simulation is based on initial velocities given by a classical molecular dynamics program package (LAMMPS). After laser excitation, the atoms move at a constant velocity. (a)-(d) show only 18 atoms for clarity. The atomic positions are shown for (a) time $=0$ fs and $T=500 \mathrm{~K}$, (b) time $=0$ fs and $T=70 \mathrm{~K}$, (c) time $=350 \mathrm{fs}$ and $T=500 \mathrm{~K}$, and (d) time $=350 \mathrm{fs}$ and $T=70 \mathrm{~K}$. The time-resolved x-ray scattering intensity for 500 (e) and for $70 \mathrm{~K}$ (f) are also shown. The full molecular movie is available online. 
by Rino et al. [16]. These potentials have previously yielded correct cohesive energy and elastic constants. They have also been used successfully to model pressure-induced phase transitions in InSb [17]. The MD simulation is classical and does not take quantum vibrational effects into account. This is a valid approximation for the two temperatures shown (500 and $70 \mathrm{~K}$ ). The initial velocities were given by the MD simulation at the time of bond breaking and we subsequently tracked each unbound atom, keeping the velocity constant. The illustrations in Figs. 1(a)-1(d) are frames from a movie which is available online. The structure of $\mathrm{InSb}$ is shown before laser excitation, and the predicted structure of laser-excited $\mathrm{InSb}$ is shown $350 \mathrm{fs}$ after laser excitation for two different temperatures. It can be seen that there is little or no disordering after $350 \mathrm{fs}$ for a sample temperature of $70 \mathrm{~K}$, while strong disordering is observed at $500 \mathrm{~K}$, as predicted by the inertial model. The rms displacement of the 4096 atoms was then used to calculate the resulting xray intensity as a function of time based on the DebyeWaller formalism [Figs. 1(e)-1(f)].

The experiment was carried out at the new short-pulse beam line "FemtoMAX" at the MAX IV synchrotron radiation facility. This incoherent, LINAC-based beam line has been described previously by Enquist et al. [17]. New undulators have recently been installed, which increased the $\mathrm{x}$-ray flux to $5 \times 10^{5}$ photons per pulse after an InSb double-crystal monochromator at a photon energy of $3.56 \mathrm{keV}$ and a bandwidth of $0.04 \%$. Grazing-incidence $\mathrm{x}$-ray diffraction measurements were made in a UHV chamber $\left(<1 \times 10^{-8}\right.$ mbar $)$ equipped with a He cryostat to allow cooling and heating of the sample. The pumpprobe experiments were carried out as a series of singleshot experiments in a crossed-beam arrangement [18]. The sample was excited with an $800 \mathrm{~nm}, 100 \mathrm{fs}$ laser at a $45^{\circ}$ angle of incidence. The $x$ rays arrived at an incident angle of $0.75^{\circ}$ with respect to the sample surface. The x-ray footprint on the sample was $18 \mathrm{~mm} \times 0.2 \mathrm{~mm}$. At this angle, the depth probed by the $\mathrm{x}$ rays $(1 / e)$ is $28 \mathrm{~nm}$. The angle of incidence was verified using a surface reflection after the sample had reached thermal equilibrium for each temperature. A schematic of the crossed-beam excitation geometry is shown in Fig. 2. The difference in the probe and pump angles corresponds to a variation in the pumpprobe delay at the sample, thus the spatial extent of the probed area along the extended dimension of the $\mathrm{x}$-ray profile can be mapped onto a time axis of the measurement. The timescale is given by

$$
t=L\left(\cos \theta_{X}-\cos \theta_{L}\right) / c,
$$

where $L$ is the position along the sample, $c$ is the speed of light in vacuum, $\theta_{X}$ denotes the incident angle of the x-ray beam on the sample surface, and $\theta_{L}$ is the corresponding angle for the laser beam. Additional technical details

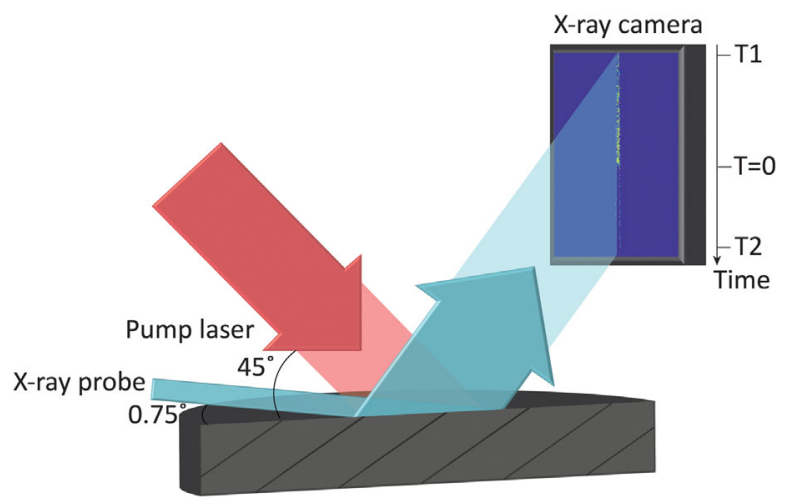

FIG. 2. Schematic of the experimental setup. Because of the difference in incident angle between the laser and the $\mathrm{x}$ rays, sample positions $\mathrm{T} 1$ and $\mathrm{T} 2$ will be exposed at different time delays, converting the spatial extent of the sample (and detector image) into a time axis.

regarding the experiment are given in the supplementary information [19]. The x-ray pulse duration was less than 200 fs. The tabulated laser penetration depth $(1 / e)$ at $800 \mathrm{~nm}$ is $100 \mathrm{~nm}$. The size of the laser beam over which the intensity was $120 \mathrm{~mJ} / \mathrm{cm}^{2} \pm 25 \%$ was $10 \mathrm{~mm} \times 0.15 \mathrm{~mm}$. The $\mathrm{x}$-ray signal that had interacted with the central $0.1 \mathrm{~mm}$ in width was used to derive the melting times. The timescale was calibrated by recording a large number of images with different settings of the relative time delay between $\mathrm{x}$-ray and laser pulses [19]. It was found to agree with Eq. (7) when the projection from the sample onto the camera was taken into account. The incident laser fluence in the evaluated data was between 90 and $150 \mathrm{~mJ} / \mathrm{cm}^{2}$, which is within the inertial regime referred to by Hillyard et al. [20]. It is close to one of the energy densities (0.259 Ry per atom pair, yielding an electron temperature of $100 \mathrm{mRy}$ ) considered by Zijlstra et al. [5]. The scattered $x$ rays were detected using an Andor iKon camera. A series of single-shot images of the 111 Bragg reflection were recorded. The temporal evolution of this reflection was determined by fitting the decrease in intensity along the temporal coordinate to a Gaussian function:

$$
I(t)=I(0) e^{-(t / \tau)^{2}}, \quad t>0 .
$$

Single shot recordings of the scattered x-ray intensity as function of time for temperatures of 500,150, and $70 \mathrm{~K}$ are shown in Fig. 3. In order to reduce and estimate the statistical error, we collected data from a series of such shots, each on a pristine part of the sample. The number of shots per series is given in Table I. The statistical analysis was performed based on the disordering time. The average and standard error were used as the measured value and experimental uncertainty for the disordering time at each temperature. In order to study the temperature dependence, data were then acquired for five different sample 


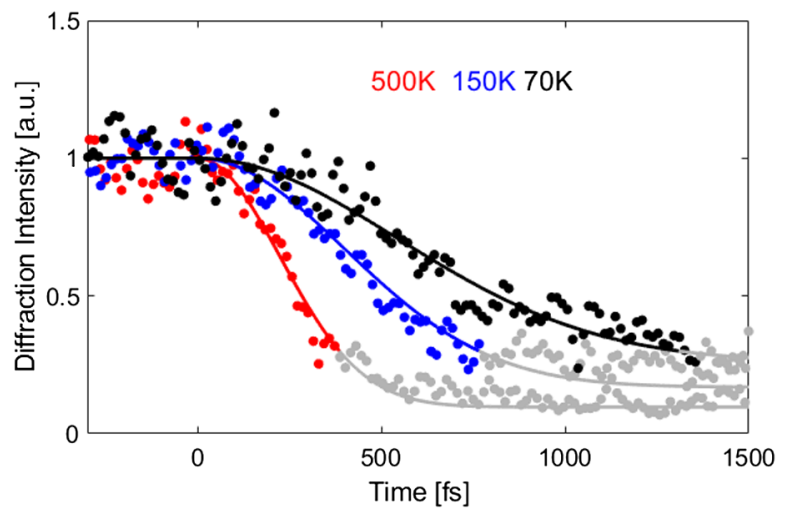

FIG. 3. Single shot recordings showing the drop in scattered $\mathrm{x}-$ ray intensity during nonthermal melting of InSb for temperatures of 500, 150, and $70 \mathrm{~K} .10-25$ curves were recorded and evaluated for each temperature. The data have been smoothed with a $60 \mathrm{fs}$ Gaussian window. The solid lines show the x-ray intensities calculated from Eqs. (1)-(5). For intensities below 30\%, the noise gives rise to large errors in the calculated displacement. We have plotted these points with lower reliability in gray.

temperatures. The disordering times and atomic velocities are given in Table I.

The laser pulse duration is $100 \mathrm{fs}$ (FWHM), divergence in the diffracted beam causes a broadening of $150 \mathrm{fs}$ (FWHM) and the duration of the x-ray pulse could be as long as $200 \mathrm{fs}$ (FWHM). The shortest measured disordering time in the study is $380 \mathrm{fs}(90 \%-10 \%)$. When convoluting simulated data with all broadening mechanisms a broadened curve would show a disordering time of $420 \mathrm{fs}$ at $500 \mathrm{~K}$. Since we just have an upper limit for the $\mathrm{x}$-ray pulse duration we have not compensated the values in Table I for experimental broadening due to the finite time resolution.

Figure 4 shows the disordering time as a function of the equilibrium sample temperature before laser excitation. Within the temperature range 35-500 K, the disordering time follows the inertial model when quantum vibrational effects are taken into account (black solid line). The dashed red line shows the disordering time when the classical

TABLE I. Measured disordering times $\left(\tau_{\text {exp }}\right)$ following nonthermal melting for several temperatures $(T)$. The calculated times $\left(t_{\bmod }\right)$ are based on the inertial model. The calculated times $\left(t_{\mathrm{cl}}\right)$ are based on the inertial model with classical vibrational energy. The value of the disordering time in the study by Lindenberg et al. [2] was calculated from the reported measured average atomic velocity.

\begin{tabular}{lcccr}
\hline \hline$T(\mathrm{~K})$ & \# of shots & $\tau_{\exp }(\mathrm{fs})$ & $t_{\text {mod }}(\mathrm{fs})$ & $t_{\mathrm{cl}}(\mathrm{fs})$ \\
\hline 500 & 18 & $410 \pm 40$ & 380 & 380 \\
300 & 10 & $470 \pm 50$ & 490 & 490 \\
150 & 21 & $760 \pm 60$ & 670 & 690 \\
70 & 10 & $960 \pm 110$ & 910 & 1010 \\
35 & 12 & $1000 \pm 80$ & 1050 & 1430 \\
300 & {$[2]$} & 530 & & 490 \\
\hline \hline
\end{tabular}

vibrational energy is used to retrieve atomic velocities. The classical description of atomic thermal vibrations is adequate down to $70 \mathrm{~K}$. Tsipenyuk [21] described different ways of measuring zero-point energy vibrations. We note that experiments such as the present one may provide a new way of probing zero-point vibrational dynamics.

Our measurements, the data from Lindenberg et al. [2] (530 fs, green diamond), the inertial model, and the calculation by Zijlstra et al. [5] are all in agreement at room temperature. The fact that the experimental results agree is not surprising, but since the two models are derived from opposite assumptions, we conclude that the convergence of the two models at room temperature is coincidental.

In order to obtain more information on the disordering process, we used the Debye-Waller equation to calculate the average atomic displacements, allowing us to track the atomic motion as a function of time. To achieve an adequate signal-to-noise ratio, we averaged all available data for each temperature. Since there was jitter between the laser and $\mathrm{x}$ ray arrival times, the different shots for each temperature had to be time stamped before the time-resolved intensities for different shots could be added. The procedure described by Lindenberg et al. was used to time stamp and average the shots [2]. The procedure consists of three steps. First, the single-shot data were fitted assuming the inertial model. The time at which the x-ray intensity was reduced by a factor two was then determined. Finally, this was used as a timing marker, and the data were shifted before summing the data from the single shots to calculate the average. Figure 5 shows the average displacement derived from our data. It can be seen that the data and the lines representing

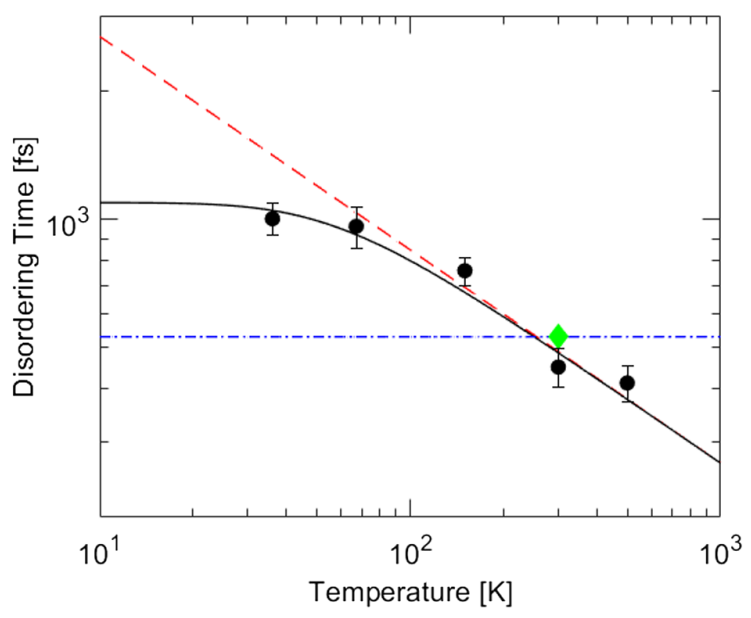

FIG. 4. The disordering time was measured at different temperatures. The experimental results are the average of a number of measurements (as given in Table I for each temperature), and the error bars show the standard error. The black solid line shows the trend predicted by the inertial model [2]. The red dashed line shows the disordering times using the classical energy. The blue dot-dashed line indicates the temperature-independent result at the value predicted by Zijlstra et al. [5]. 


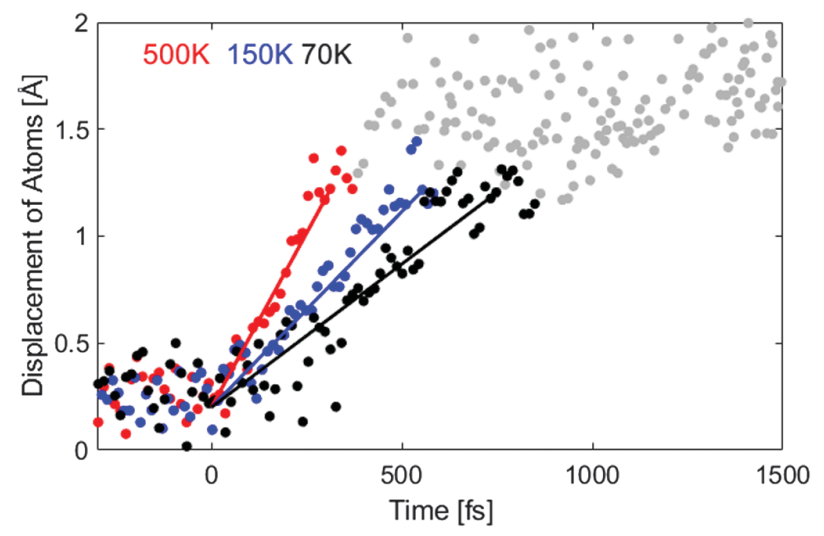

FIG. 5. Atomic average displacement for temperatures of 500, 150, and $70 \mathrm{~K}$, calculated using Eqs. (1)-(5) for the average of single-shot measurements. The number of shots for each temperature is given in Table I. The atomic motion was tracked up to $1.2 \AA$, corresponding to a decrease in the initial x-ray intensity of the 111 Bragg reflection of $30 \%$. For intensities below this level, the noise gives rise to large errors in the calculated displacement. We have plotted these points with lower reliability in gray. The solid lines are calculated from the initial velocity given by thermodynamic equilibrium dynamics at the respective temperature.

inertial motion are in excellent agreement. This can be expected since the disordering time is in agreement with the inertial model.

We have shown experimentally that the atomic motion during a phase transition depends on the initial temperature of the material being studied. This leads to the conclusion that thermal equilibrium dynamics must be considered when predicting the timescale for ultrafast phase transitions. The only parameters used to predict the disordering time are the atomic masses, the temperature, and the Debye temperature. Thus it can be expected that thermal equilibrium dynamics will play a role in determining the timescale of a photo-induced phase transition, regardless of the type of material, and how much energy is deposited in the electron system during the excitation process. The inertial model, which allows determination of the initial atomic velocities, can be used to estimate the timescales of phase transitions in solid materials. This approach was used in a recent study of a phase transition in $\mathrm{VO}_{2}$ by Wall et al. [22], who interpreted reordering timescales in terms of inertial motion. Including thermal equilibrium dynamics may be important but it is not sufficient. It is known from previous studies that the potential surface can accelerate unbound atoms, and that the acceleration depends on the type of material and the amount of energy transferred from the optical excitation pulse to the electron system. This has been observed, for example, in $\mathrm{Bi}$ [23] and InSb [20]. Previous theoretical studies $[4,5,24]$ have shown that bondbreaking processes such as nonthermal melting can be accurately described by $a b$ initio models predicting the potential landscape following bond breaking. However, the present study shows that such calculations do not tell the whole story. Such calculations must be augmented with MD simulations in which thermal equilibrium dynamics is included. Lian et al. recently performed a calculation including initial atomic velocities [13] for the description of laser-induced nonthermal melting. However, other aspects of their work have been disputed [14]. To directly compare computed, nonequilibrium potentials to the results of experiments, the initial velocities should be estimated to determine whether measurements should be performed on cryogenically cooled samples. Coherent control of phase transitions in solids has been the ambition since it became possible to carry out ultrafast, time-resolved structurally sensitive measurements. Recent reports have demonstrated significant steps in this direction $[25,26]$. The present study highlights one of the difficulties by showing that random thermal effects can be significant, but can be reduced to the zero-point limit by cooling the material under investigation.

The authors would like to thank the Swedish Research Council (VR, Grant No. 2014-4732), Stiftelsen Olle Engkvist byggmästare (Grant No. 1041513), and the X-PROBE Marie Skłodowska-Curie ITN within Horizon 2020 (No. 637295) for financial support. The FemtoMAX beam line was funded by the Knut and Alice Wallenberg Foundation (KAW, No. 2010.0098) and 12 Swedish Universities: Lund University, Chalmers University of Technology, Karlstad University, Karolinska Institutet, KTH Royal Institute of Technology, Linköping University, Luleå University of Technology, Stockholm University, Swedish University of Agricultural Sciences, Umeå University, University of Gothenburg, and Uppsala University.

*jorgen.larsson@fysik.lth.se

[1] P. Zalden et al., Science 364, 1062 (2019).

[2] A. M. Lindenberg et al., Science 308, 392 (2005).

[3] M. Z. Mo et al., Science 360, 1451 (2018).

[4] T. Zier, E. S. Zijlstra, and M. E. Garcia, Phys. Rev. Lett. 116, 153901 (2016).

[5] E. S. Zijlstra, J. Walkenhorst, and M. E. Garcia, Phys. Rev. Lett. 101, 135701 (2008).

[6] R. Ernstorfer, M. Harb, C. T. Hebeisen, G. Sciaini, T. Dartigalongue, and R. J. D. Miller, Science 323, 1033 (2009).

[7] P. Stampfli and K. H. Bennemann, Phys. Rev. B 49, 7299 (1994).

[8] J. A. Van Vechten, R. Tsu, F. W. Saris, and D. Hoonhout, Phys. Lett. 74A, 417 (1979).

[9] C. V. Shank, R. Yen, and C. Hirlimann, Phys. Rev. Lett. 50, 454 (1983).

[10] C. V. Shank, R. Yen, and C. Hirlimann, Phys. Rev. Lett. 51, 900 (1983).

[11] A. Rousse et al., Nature (London) 410, 65 (2001).

[12] K. Sokolowski-Tinten, C. Blome, C. Dietrich, A. Tarasevitch, M. Horn von Hoegen, D. von der Linde, A. Cavalleri, J. Squier, and M. Kammler, Phys. Rev. Lett. 87, 225701 (2001). 
[13] C. Lian, S. B. Zhang, and S. Meng, Phys. Rev. B 94, 184310 (2016).

[14] T. Zier, M. E. Garcia, and P. L. Silvestrelli, Phys. Rev. B 99, 066301 (2019).

[15] K. Nakamura and T. Mohri, Model Simul. Mater. Sci. 1, 143 (1993).

[16] J. Bang, Y. Y. Sun, X. Q. Liu, F. Gao, and S. B. Zhang, Phys. Rev. Lett. 117, 126402 (2016).

[17] H. Enquist et al., J. Synchrotron Radiat. 25, 570 (2018).

[18] O. Synnergren, M. Harbst, T. Missalla, J. Larsson, G. Katona, R. Neutze, and R. Wouts, Appl. Phys. Lett. 80, 3727 (2002).

[19] See Supplemental Material at http://link.aps.org/supplemental/ 10.1103/PhysRevLett.124.105701 for molecular movie showing the disordering illustrated in Fig. 1 and details about calibration of time and incidence angle.

[20] P. B. Hillyard et al., Phys. Rev. Lett. 98, 125501 (2007).

[21] Y. M. Tsipenyuk, Phys. Usp. 55, 796 (2012).

[22] S. Wall et al., Science 362, 572 (2018).

[23] G. Sciaini, M. Harb, S. G. Kruglik, T. Payer, C. T. Hebeisen, F.-J. Meyer zu Heringdorf, M. Yamaguchi, M. Horn-von Hoegen, R. Ernstorfer, and R. J. D. Miller, Nature (London) 458, 56 (2009).

[24] P. Stampfli and K. H. Bennemann, Appl. Phys. A 60, 191 (1995).

[25] E. J. Sie et al., Nature (London) 565, 61 (2019).

[26] T. F. Nova, A. S. Disa, M. Fechner, and A. Cavalleri, Science 364, 1075 (2019). 